\title{
EFFECTS OF PULSED DYE LASER ON THE BILIARY WALL OF THE DOG
}

P. GAGNON, T. PONCHON, A. NAOURI, F. BERGER and L. HENRY

Dept of Digestive Diseases, Hôpital E. Herriot, Lyon, France

Pulsed dye laser might be useful for biliary lithotripsy. Because of its inherent characteristics, this laser is thought to be safe for the biliary wall. The purpose of this in vivo experiment was to study the safety of pulsed dye laser on the gallbladder and common bile duct wall of the dog. Methods: Six beagle dogs weighing about $15 \mathrm{Kg}$ were used. For each dog, the following protocol was applied: a) General anesthesia and laparotomy; b) Gallbladder: 1-Longitudinal incision, 2-Fixation of a specially designed device on the mucosa, through which the fibre was inserted, in order to keep a constant contact between the fibre and the mucosa despite breathing movements, 3-Application of the tibre perpendicular and in gentle contact with the mucosa, and gallbladder immersion in saline, 4-Laser firing on 8 different points of the mucosa, each point corresponding to a different number of pulses fired: $0,10,50,100,250$, 500,1000 , and $3000,5-P u l s e$ rate: $5 \mathrm{~Hz}, 6-$ Three levels of energy per pulse were studied using 2 dogs for each level: 60,90 , and $120 \mathrm{~mJ} /$ pulse respectively, 7- Identification of each firing point with India ink and gallbladder ablation for histologic study. c) Common bile duct: The same protocol was used except that only 4 firing points were studied: 10,100 , 1000 and 3000 respectively.

Results: In no case was a biliary wall perforation noted. Hemorrhagic infiltrations and lesions of coagulation necrosis were observed. Lesions were limited to the epithelium or extended transmurally; the depth increased with increasing energy/pulse or number of pulses per point. The diameter of the lesions was at least equal to the fibre diameter but was less than $2.0 \mathrm{~mm}$. For the gallbladder, a transmural necrosis which could potentially lead to perforation was observed when firing $>250$ pulses at $120 \mathrm{~mJ},>500$ pulses at $90 \mathrm{~mJ}$, and $>1000$ pulses at $60 \mathrm{~mJ}$. For the common bile duct, it was observed with $>100$ pulses at $120 \mathrm{~mJ}$ and with $>1000$ pulses at 60 and $90 \mathrm{~mJ}$. In these experimental conditions, these number of pulses should be considered the safety limits.

conclusion: When firing perpendicular and in contact with the mucosa, pulsed dye laser may result in significant histological biliary wall lesions; In clinical situations, this laser could be safely used under direct vision or even under fluoroscopy provided that the safety limits of firing as defined in this experiment are respected. 